\title{
Soft $\omega^{*}$-Paracompactness in Soft Topological Spaces
}

Samer Al Ghour

Department of Mathematics and Statistics, Jordan University of Science and Technology, Irbid, Jordan

\begin{abstract}
In this study, we introduce a new concept in soft topological spaces, namely, soft $\omega^{*}$ paracompactness, and we provide characterizations thereof. Its connection with other related concepts is also studied. In particular, we show that soft $\omega^{*}$-paracompactness and soft paracompactness are independent of each other. In addition, we study the soft $\omega^{*}$-paracompactness of the soft topological space generated by an indexed family of $\omega^{*}$-paracompact topological spaces.
\end{abstract}

Keywords: $\omega$-open sets, $\omega^{*}$-paracompact, Soft paracompact, Generated soft topology

\section{Introduction}

Throughout this paper, we use the notions and terminology in [1] and [2]; moreover, TS and STS stand for "topological space" and "soft topological space," respectively. Recently, classical methods have been applied to several problems in various fields, such as engineering, social sciences, and medical sciences. Soft sets were defined by Molodtsov [3], and they have found numerous applications. Let $X$ be a universal set and $A$ be a set of parameters. A soft set over $X$ relative to $A$ is a function $G: A \longrightarrow \mathcal{P}(X)$. The family of all soft sets over $X$ relative to $A$ is denoted by $S S(X, A)$. If $G \in S S(X, A)$ is such that $G(a)$ is a countable set for all $a \in X$, then $G$ is called a countable soft set. The family of all members of $S S(X, A)$ that are countable is denoted by $C S S(X, A)$. We denote the null soft set and the absolute soft set as $0_{A}$ and $1_{A}$, respectively. Soft topological spaces were defined in [4] as follows: A triplet $(X, \tau, A)$, where $\tau \subseteq S S(X, A)$, is called an STS if $0_{A}$ and $1_{A} \in \tau$, and $\tau$ is closed

(C)This is an Open Access article distributed under the terms of the Creative Commons Attribution Non-Commercial License http://creativecommons.org/licenses/by-nc/ 3.0/) which permits unrestricted noncommercial use, distribution, and reproduction in any medium, provided the original work is properly cited. under finite soft intersections and arbitrary soft unions. For an STS $(X, \tau, A)$, the members of $\tau$ are called soft open sets, and their soft complements are called soft closed sets. Several topological concepts have been extended to the context of STSs [1, 2, 5- 28]. The concept of $\omega$-open set was introduced in [29] as a weaker form of an open set as follows: Let $(X, \Im)$ be a TS and let $U \subseteq X$; then, $U$ is called $\omega$-open if for every $x \in U$, there is an open set $V$ and a countable subset $C \subseteq X$ such that $x \in V-C \subseteq U$.

The complement of an $\omega$-open set in $(X, \Im)$ is called an $\omega$-closed set. The family of all $\omega$-open sets in $(X, \Im)$ is denoted by $\Im_{\omega}$. It is known that $\Im_{\omega}$ forms a topology on $X$ that contains $\Im$. The study of $\omega$-open sets remains an area that attracts considerable attention [30-35]. Using $\omega$-open sets, the concept of $\omega^{*}$-paracompactness was defined and studied in ordinary TSs [36]. In [2], the concept of $\omega$-openness was extended to include STSs as follows: Let $(X, \tau, A)$ be an STS, and let $G \in S S(X, A)$; then, $G$ is called a soft $\omega$-open set if for all 
$a_{x} \widetilde{\in} H$, there exist $F \in \tau$ and $H \in C S S(X, A)$ such that $a_{x}$ $\widetilde{\in} F-H \widetilde{\subseteq} G$. The collection of all soft $\omega$-open sets in $(X, \tau, A)$ is denoted by $\tau_{\omega}$. It was proved in [2] that $\left(X, \tau_{\omega}, A\right)$ is an STS with $\tau \subseteq \tau_{\omega}$.

In this study, using soft $\omega$-open sets, we introduce a new concept in STSs, namely, $\omega^{*}$-paracompactness, which extends $\omega^{*}$-paracompactness in ordinary topological spaces. As symmetries, we provide characterizations of this concept. We also study the connection of soft $\omega^{*}$-paracompactness with other related topological concepts. In particular, we show that soft $\omega^{*}$-paracompactness and soft paracompactness are independent of each other. Moreover, we study the soft $\omega^{*}$-paracompactness of the STS generated by an indexed family of $\omega^{*}$-paracompact TSs.

\section{Preliminaries}

Herein, we recall several related definitions and results.

Definition 2.1. Let $(X, \Im)$ be a TS.

(a) The set of all covers of $(X, \Im)$ is denoted by $C(X, \Im)$ and defined by

$$
C(X, \Im)=\{\mathcal{B} \subseteq \mathcal{P}(X): \cup\{B: B \in \mathcal{B}\}=X\} .
$$

(b) The set of all open covers of $(X, \Im)$ is denoted by $O C(X$, $\Im)$ and defined by

$$
O C(X, \Im)=\{\mathcal{B} \subseteq \Im: \cup\{B: B \in \mathcal{B}\}=X\}
$$

We recall that if $\mathcal{A}, \mathcal{B} \in C(X, \Im)$, where $(X, \Im)$ is a TS, then $\mathcal{A}$ is called a refinement of $\mathcal{B}$ (denoted as $\mathcal{A} \preceq \mathcal{B}$ ) if for each $A \in \mathcal{A}$, there is $B \in \mathcal{B}$ such that $A \subseteq B$. Moreover, let $(X, \Im)$ be a TS and $\mathcal{A} \subseteq \mathcal{P}(X)$; then, $\mathcal{A}$ is called locally finite in $(X, \Im)$ if for each $x \in X$, there is $U \in \Im$ such that $x \in U$ and $\{A \in \mathcal{A}: U \cap A \neq \emptyset\}$ is finite.

Definition 2.2 ([36]). A TS $(X, \Im)$ is called $\omega^{*}$-paracompact if for every $\mathcal{B} \in O C\left(X, \Im_{\omega}\right)$, there is $\mathcal{A} \in O C\left(X, \Im_{\omega}\right)$ such that $\mathcal{A}$ is locally finite in $(X, \Im)$, and $\mathcal{A}$ is a refinement of $\mathcal{B}$.

Definition 2.3. Let $(X, \tau, A)$ be an STS.

(a) The set of all soft covers of $(X, \tau, A)$ is denoted by $S C(X, \tau, A)$ and defined by

$$
S C(X, \tau, A)=\left\{\mathcal{H} \subseteq S S(X, A): \widetilde{\cup}\{H: H \in \mathcal{H}\}=1_{A}\right\} .
$$

(b) The set of all soft open covers of $(X, \tau, A)$ is denoted by $\operatorname{SOC}(X, \tau, A)$ and defined by

$$
S O C(X, \tau, A)=\left\{\mathcal{H} \subseteq \tau: \widetilde{\cup}\{H: H \in \mathcal{H}\}=1_{A}\right\} .
$$

(c) The set of all soft closed covers of $(X, \tau, A)$ is denoted by $\operatorname{SCC}(X, \Im)$ and defined by

$$
S C C(X, \tau, A)=\left\{\mathcal{H}: \mathcal{H} \subseteq \tau^{c}: \widetilde{\cup}\{H: H \in \mathcal{H}\}=1_{A}\right\} .
$$

Definition $2.4([37 \mid)$. Let $(X, \tau, A)$ be an STS and $\mathcal{H}, \mathcal{M} \subseteq$ $S S(X, A)$.

(a) $\mathcal{H}$ is called soft locally finite in $(X, \tau, A)$ if for each $a_{x} \in S P(X, A)$, there is $F \in \tau$ such that $a_{x} \widetilde{\in} F$, and $\left\{H \in \mathcal{H}: F \widetilde{\cap} H \neq 0_{A}\right\}$ is finite.

(b) $\mathcal{M}$ is called a soft refinement of $\mathcal{H}$ (denoted as $\mathcal{M} \preceq \mathcal{H}$ ) if for each $M \in \mathcal{M}$, there is $H \in \mathcal{H}$ such that $M \widetilde{\simeq} H$.

Definition 2.5 ([37]). An STS $(X, \tau, A)$ is called soft paracompact if for every $\mathcal{H} \in S O C(X, \tau, A)$, there is $\mathcal{M} \in S O C$ $(X, \tau, A)$ such that $\mathcal{M}$ is soft locally finite in $(X, \tau, A)$ and $\mathcal{M}$ $\preceq \mathcal{H}$.

Definition 2.6 ([4] ). An STS $(X, \tau, A)$ is called soft regular if for every $F \in \tau^{c}$ and $a_{x} \widetilde{\notin} F$, there exist $U, V \in \tau$ such that $a_{x} \widetilde{\in} U, F \widetilde{\subseteq} V$, and $U \widetilde{\cap} V=0_{A}$.

Proposition $2.7([4])$. An STS $(X, \tau, A)$ is soft regular if for every $V \in \tau$ and $a_{x} \widetilde{\in} U$, there exists $V \in \tau$ such that $a_{x} \widetilde{\in} V$ $\widetilde{\subseteq} C l_{\tau}(V) \widetilde{\subseteq} U$.

Proposition $2.8([37])$. Let $(X, \tau, A)$ be an STS, where $A=\{a\}$. Then, $(X, \tau, A)$ is soft paracompact if and only if the $\operatorname{TS}\left(X, \tau_{a}\right)$ is paracompact.

Proposition 2.9 ([2]). Let $(X, \tau, A)$ be an STS. Then, for all $a \in A$, we have $\left(\tau_{a}\right)_{\omega}=\left(\tau_{\omega}\right)_{a}$.

Proposition 2.10 ([2]). Let $X$ be an initial universe, let $A$ be a set of parameters, and let $\left\{\Im_{a}: a \in A\right\}$ be an indexed family of topologies on $X$. Then, $\left(\bigoplus_{a \in A} \Im_{a}\right)_{\omega}=\bigoplus_{a \in A}\left(\Im_{a}\right)_{\omega}$.

\section{Soft $\omega^{*}$-Paracompactness}

This study is primarily concerned with the following concept.

Definition 3.1. An STS $(X, \tau, A)$ is called soft $\omega^{*}$-paracompact if for every $\mathcal{H} \in S O C\left(X, \tau_{\omega}, A\right)$, there is $\mathcal{M} \in$ $\operatorname{SOC}\left(X, \tau_{\omega}, A\right)$ such that $\mathcal{M}$ is soft locally finite in $(X, \tau, A)$ and $\mathcal{M} \preceq \mathcal{H}$.

The following three Lemmas are used in Theorem 3.6 below.

Lemma 3.2. If $\mathcal{H}$ is soft locally finite in $(X, \tau, A)$, then $\left\{C l_{\tau_{\omega}}(H): H \in \mathcal{H}\right\}$ is soft locally finite in $(X, \tau, A)$.

Proof. Let $a_{x} \in S P(X, A)$; then, there is $F \in \tau$ such that $a_{x} \in F$, and $\left\{H \in \mathcal{H}: F \widetilde{\cap} H \neq 0_{A}\right\}$ is finite.

Claim. $\left\{H \in \mathcal{H}: F \widetilde{\cap} C l_{\tau_{\omega}}(H) \neq 0_{A}\right\} \subseteq$ $\left\{H \in \mathcal{H}: F \widetilde{\cap} H \neq 0_{A}\right\}$. 
Proof of Claim. We assume that $F \widetilde{\cap} C l_{\tau_{\omega}}(H) \neq 0_{A}$ for some $H \in \mathcal{H}$. We select $a_{x} \widetilde{\in} F \widetilde{\cap} C l_{\tau_{\omega}}(H) \widetilde{\widetilde{\subseteq}} F \widetilde{\cap} C l_{\tau}(H)$; then, $a_{x} \widetilde{\in} F \widetilde{\cap} C l_{\tau}(H)$, and thus $F \widetilde{\cap} H \neq 0_{A}$.

Lemma 3.3. If $\mathcal{H}$ is soft locally finite in $(X, \tau, A)$, then $\mathcal{H}$ is soft locally finite in $\left(X, \tau_{\omega}, A\right)$.

Proof. Let $a_{x} \in S P(X, A)$; then, there is $F \in \tau \subseteq \tau_{\omega}$ such that $a_{x} \in F$, and $\left\{H \in \mathcal{H}: F \widetilde{\cap} H \neq 0_{A}\right\}$ is finite.

The following example shows that the converse of the implication in Lemma 3.3 is not true in general.

Example 3.4. Let $X=\mathbb{N}, \tau=\left\{0_{A}, 1_{A}\right\}, A=\mathbb{R}$, and $\mathcal{H}=S P(X, A)$. Then, $\mathcal{H}$ is soft locally finite in $\left(X, \tau_{\omega}, A\right)$, but it is not soft locally finite in $(X, \tau, A)$.

Lemma 3.5. If $\mathcal{H}$ is soft locally finite in $(X, \tau, A)$, then

$$
\widetilde{\cup}\left\{C l_{\tau_{\omega}}(H): H \in \mathcal{H}\right\}=C l_{\tau_{\omega}}(\widetilde{\cup}\{H: H \in \mathcal{H}\}) .
$$

Proof. We assume that $\mathcal{H}$ is soft locally finite in $(X, \tau, A)$. By Lemma $3.3, \mathcal{H}$ is soft locally finite in $\left(X, \tau_{\omega}, A\right)$. Thus, by applying Proposition 5.2 in [37], we obtain the result.

The following provides two characterizations of soft $\omega^{*}$ paracompact STSs.

Theorem 3.6. Let $(X, \tau, A)$ be an STS so that $\left(X, \tau_{\omega}, A\right)$ is soft regular. Then, the following are equivalent:

(a) $(X, \tau, A)$ is soft $\omega^{*}$-paracompact.

(b) For every $\mathcal{H} \in S O C\left(X, \tau_{\omega}, A\right)$, there is $\mathcal{M} \in S C\left(X, \tau_{\omega}\right.$, $A$ ) such that $\mathcal{M}$ is soft locally finite in $(X, \tau, A)$ and $\mathcal{M} \preceq \mathcal{H}$.

(c) For every $\mathcal{H} \in S O C\left(X, \tau_{\omega}, A\right)$, there is $\mathcal{M} \in S C C(X$, $\left.\tau_{\omega}, A\right)$ such that $\mathcal{M}$ is soft locally finite in $(X, \tau, A)$ and $\mathcal{M} \preceq$ $\mathcal{H}$.

Proof. (a) $\Longrightarrow$ (b): Obvious.

(b) $\Longrightarrow$ (c): We assume that $(X, \tau, A)$ is soft $\omega^{*}$-paracompact, and let $\mathcal{H} \in S O C\left(X, \tau_{\omega}, A\right)$. For every $a_{x} \in S P(X, A)$, we select $H_{a_{x}} \in \mathcal{H}$ such that $a_{x} \widetilde{\in} H_{a_{x}}$. As $\left(X, \tau_{\omega}, A\right)$ is soft regular, by Proposition 2.7, for every $a_{x} \in S P(X, A)$, there is $N_{a_{x}} \in \tau$ such that $a_{x} \widetilde{\in} N_{a_{x}} \widetilde{\subseteq} C l_{\tau_{\omega}}\left(N_{a_{x}}\right) \widetilde{\subseteq} H_{a_{x}}$. Let $\mathcal{N}=\left\{N_{a_{x}}: a_{x} \in S P(X, A)\right\}$; then, $\mathcal{N} \in S O C\left(X, \tau_{\omega}, A\right)$. As $(X, \tau, A)$ is soft $\omega^{*}$-paracompact, there is $\mathcal{M} \in S O C\left(X, \tau_{\omega}\right.$, $A$ ) such that $\mathcal{M}$ is soft locally finite in $(X, \tau, A)$ and $\mathcal{M} \preceq \mathcal{N}$. Let $\mathcal{M}_{1}=\left\{C l_{\tau_{\omega}}(M): M \in \mathcal{M}\right\}$. Then, $\mathcal{M}_{1} \in S C C\left(X, \tau_{\omega}\right.$, $A$ ), and by Lemma 3.2, $\mathcal{M}_{1}$ is soft locally finite.

Claim (i). $\mathcal{M}_{1} \preceq \mathcal{H}$.

Proof of Claim. Let $C l_{\tau_{\omega}}(M) \in \mathcal{M}_{1}$, where $M \in \mathcal{M}$. As $\mathcal{M} \preceq \mathcal{N}$, there is $a_{x} \in S P(X, A)$ such that $M \widetilde{\subseteq} N_{a_{x}}$, and thus $C l_{\tau_{\omega}}(M) \widetilde{\subseteq} C l_{\tau_{\omega}}\left(N_{a_{x}}\right) \widetilde{\subseteq} H_{a_{x}}$ with $H_{a_{x}} \in \mathcal{H}$. It follows that $\mathcal{M}_{1} \preceq \mathcal{H}$.

(c) $\Longrightarrow$ (a): Let $\mathcal{H} \in S O C\left(X, \tau_{\omega}, A\right)$. By (c), there is $\mathcal{L} \in$ $S C C\left(X, \tau_{\omega}, A\right)$ such that $\mathcal{L}$ is soft locally finite in $(X, \tau, A)$ and $\mathcal{L} \preceq \mathcal{H}$. As $\mathcal{L}$ is soft locally finite, for each $a_{x} \in S P(X, A)$, there is $N_{a_{x}} \in \tau$ such that $a_{x} \widetilde{\in} N_{a_{x}}$, and $\left\{L \in \mathcal{L}: N_{a_{x}} \widetilde{\cap} L \neq\right.$ $\left.0_{A}\right\}$ is finite. Let $\mathcal{N}=\left\{N_{a_{x}}: a_{x} \in S P(X, A)\right\}$. Then, $\mathcal{N} \in$ $\operatorname{SOC}(X, \tau, A) \subseteq S O C\left(X, \tau_{\omega}, A\right)$, and by (c), there is $\mathcal{G} \in$ $\operatorname{SCC}\left(X, \tau_{\omega}, A\right)$ such that $\mathcal{G}$ is soft locally finite in $(X, \tau, A)$ and $\mathcal{G} \preceq \mathcal{N}$. As $\mathcal{L} \preceq \mathcal{H}$, for every $L \in \mathcal{L}$, there is $H_{L} \in$ $\mathcal{H}$ such that $L \widetilde{\subseteq} H_{L}$. For every $L \in \mathcal{L}$, let $K_{L}=1_{A}-$ $\widetilde{\cup}\left\{G \in \mathcal{G}: G \widetilde{\cap} L=0_{A}\right\}$ and $M_{L}=K_{L} \widetilde{\cap} H_{L}$.

Claim (ii). (1) $\left\{K_{L}: L \in \mathcal{L}\right\}$. $\subseteq \tau_{\omega}$.

(2) For every $L \in \mathcal{L}$, we have $L \widetilde{\subseteq} K_{L}$.

(3) For every $L \in \mathcal{L}$ and $G \in \mathcal{G}, K_{L} \widetilde{\cap} G \neq 0_{A}$ if and only if $L \widetilde{\cap} G \neq 0_{A}$.

(4) $\left\{K_{L}: L \in \mathcal{L}\right\}$ is soft locally finite.

(5) $\left\{M_{L}: L \in \mathcal{L}\right\} \in S O C\left(X, \tau_{\omega}, A\right)$.

(6) $\left\{M_{L}: L \in \mathcal{L}\right\} \preceq \mathcal{H}$.

(7) $\left\{M_{L}: L \in \mathcal{L}\right\}$ is soft locally finite.

Proof of Claim. 1. Follows from Lemma 3.5.

2. We assume toward a contradiction that there are $L_{0} \in \mathcal{L}$ and $a_{x} \in S P(X, \tau)$ such that $a_{x} \tilde{\in} L_{0} \widetilde{\cap}\left(\widetilde{\cup}\left\{G \in \mathcal{G}: G \widetilde{\cap} L_{0}=\right.\right.$ $\left.\left.0_{A}\right\}\right)$. Then, $a_{x} \widetilde{\in} L_{0}$ and $a_{x} \widetilde{\in}\left(\widetilde{\cup}\left\{G \in \mathcal{G}: G \widetilde{\cap} L_{0}=0_{A}\right\}\right)$. As $a_{x} \widetilde{\in}\left(\widetilde{\cup}\left\{G \in \mathcal{G}: G \widetilde{\cap} L_{0}=0_{A}\right\}\right)$, there is $G_{x} \in \mathcal{G}$ such that $G_{x} \widetilde{\cap} L_{0}=0_{A}$ and $a_{x} \widetilde{\in} G_{x}$. However, $a_{x} \sim \in G_{x} \widetilde{\cap} L_{0}$, which is a contradiction.

3. Necessity. Let $L \in \mathcal{L}$ and $G \in \mathcal{G}$ such that $K_{L} \widetilde{\cap} G \neq 0_{A}$, and we assume toward a contradiction that $L \widetilde{\cap} G=0_{A}$. We select $a_{x} \sim \in K_{L} \widetilde{\cap} G$. Then, $a_{x} \widetilde{\in} K_{L}$ and $a_{x} \widetilde{\in} G$. As $L \widetilde{\cap}$ $G=0_{A}$ and $a_{x} \widetilde{\in} G$, we have $a_{x} \widetilde{\in} \widetilde{\cup}\left\{G \in \mathcal{G}: G \widetilde{\cap} L=0_{A}\right\}$ and so $a_{x} \widetilde{\notin} K_{L}$, which is a contradiction.

Sufficiency. Let $L \in \mathcal{L}$ and $G \in \mathcal{G}$ such that $L \widetilde{\cap} G \neq 0_{A}$. By (2), $L \widetilde{\subseteq} K_{L}$, and therefore $L \widetilde{\cap} G \widetilde{\subseteq} K_{L} \widetilde{\cap} G$. It follows that $K_{L} \widetilde{\cap} G \neq 0_{A}$.

4. Let $a_{x} \in S P(X, \tau)$. As $\mathcal{G}$ is soft locally finite, there is $S \in \tau$ such that $a_{x} \widetilde{\in} S$, and $\left\{G \in \mathcal{G}: G \widetilde{\cap} S \neq 0_{A}\right\}$ is finite; let $\left\{G \in \mathcal{G}: G \widetilde{\cap} S \neq 0_{A}\right\}=\left\{G_{1}, G_{2}, \ldots, G_{n}\right\}$. We assume that for some $L \in \mathcal{L}$, we have $K_{L} \widetilde{\cap} S \neq 0_{A}$. We select $b_{y} \widetilde{\in} K_{L}$ $\widetilde{\cap} S$. As $b_{y} \widetilde{\in} 1_{A}=\widetilde{\cup}\{G: G \in \mathcal{G}\}$, there is $G \in \mathcal{G}$ such that $b_{y} \widetilde{\in} G$. As $b_{y} \widetilde{\in} K_{L}$, by (3), we have $G \widetilde{\cap} L \neq 0_{A}$. As $b_{y} \widetilde{\in}$ $G \widetilde{\cap} S$, we have $G=G_{i}$ for some $i=1,2, \ldots, n$. As $\mathcal{G} \preceq \mathcal{N}$, for every $i=1,2, \ldots, n$, there is $\left(a_{i}\right)_{x_{i}}$ such that $G_{i} \widetilde{\subseteq} N_{\left(a_{i}\right)_{x_{i}}}$. Therefore, $\left\{L \in \mathcal{L}: K_{L} \widetilde{\cap} S \neq 0_{A}\right\}$ is finite. It follows that $\left\{K_{L}: L \in \mathcal{L}\right\}$ is soft locally finite.

5. Let $L \in \mathcal{L}$. As $H_{L} \in \mathcal{H} \subseteq \tau_{\omega}$, and by (1), we have $K_{L} \in$ 
$\tau_{\omega}$, it follows that $M_{L}=K_{L} \widetilde{\cap} H_{L} \in \tau_{\omega}$. However, by (2) $L$. Moreover, $L \widetilde{\subseteq} H_{L}$, and by (2), we have $L \widetilde{\subseteq} K_{L}$; accordingly, $L \widetilde{\subseteq} M_{L}$. It follows that $\left\{M_{L}: L \in \mathcal{L}\right\} \subseteq \tau_{\omega}$ and $1_{A}=\widetilde{U}$ $\{L: L \in \mathcal{L}\} \widetilde{\simeq} \widetilde{\cup}\left\{M_{L}: L \in \mathcal{L}\right\}$. Therefore, $\left\{M_{L}: L \in \mathcal{L}\right\}$ $\in \operatorname{SOC}\left(X, \tau_{\omega}, A\right)$.

6. Let $L \in \mathcal{L}$. Then, $M_{L}=K_{L} \widetilde{\cap} H_{L} \widetilde{\subseteq} H_{L}$ with $H_{L} \in \mathcal{H}$.

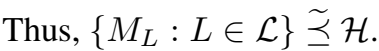

7. This follows directly from (4).

The following characterization of soft paracompact STSs is used in the proof of Theorem 3.8.

Proposition 3.7. Let $(X, \tau, A)$ be an STS, and let $\mathcal{B}$ be a soft base of $\tau$. Then, $(X, \tau, A)$ is soft paracompact if and only if for every $\mathcal{H} \in S O C(X, \tau, A)$ with $\mathcal{H} \subseteq \mathcal{B}$, there is $\mathcal{M} \in$ $S O C(X, \tau, A)$ such that $\mathcal{M}$ is soft locally finite in $(X, \tau, A)$ and $\mathcal{M} \preceq \mathcal{H}$.

Proof. Straightforward.

Theorem 3.8. Let $X$ be an initial universe, and let $A$ be a set of parameters. Moreover, let $\left\{\Im_{a}: a \in A\right\}$ be an indexed family of topologies on $X$. Then, $\left(X, \bigoplus_{a \in A} \Im_{a}, A\right)$ is soft paracompact if and only if $\left(X, \Im_{a}\right)$ is paracompact for all $a \in$ A.

Proof. Necessity. We assume that $\left(X, \bigoplus_{a \in A} \Im_{a}, A\right)$ is soft paracompact. Let $b \in A$. To show that $\left(X, \Im_{b}\right)$ is paracompact, let $\mathcal{U} \subseteq O C\left(X, \Im_{b}\right)$. We set $\mathcal{H}=\left\{b_{U}: U \in \mathcal{U}\right\} \cup$ $\left\{a_{X}: a \in A-\{b\}\right\}$. Then, $\mathcal{H} \in \mathcal{S O C}\left(X, \bigoplus_{a \in A} \Im_{a}, A\right)$. As $\left(X, \bigoplus_{a \in A} \Im_{a}, A\right)$ is soft paracompact, there is $\mathcal{M} \in \mathcal{S O C}(X$, $\left.\bigoplus_{a \in A} \Im_{a}, A\right)$ such that $\mathcal{M}$ is soft locally finite in $\left(X, \bigoplus_{a \in A} \Im_{a}, A\right)$ and $\mathcal{M} \preceq \mathcal{H}$. Let $\mathcal{M}_{b}=\{M(b): M \in \mathcal{M}\}$.

Claim. (1) $\mathcal{M}_{b} \in O C\left(X, \Im_{b}\right)$.

(2) $\mathcal{M}_{b}$ is locally finite in $\left(X, \Im_{b}\right)$.

(3) $\mathcal{M}_{b} \preceq \mathcal{U}$.

Proof of Claim. 1. As $\mathcal{M} \subseteq \bigoplus_{a \in A} \Im_{a}$, by the definition of $\Im_{b}$, we have $\mathcal{M}_{b} \subseteq \Im_{b}$. As $\mathcal{M} \in \mathcal{S O C}\left(X, \bigoplus_{a \in A} \Im_{a}, A\right)$, we have $\widetilde{\cup}\{M: M \in \mathcal{M}\}=1_{A}$, and thus $(\widetilde{\cup}\{M: M \in \mathcal{M}\})(b)=$ $\cup\{M(b): M \in \mathcal{M}\}=X$. Therefore, $\mathcal{M}_{b} \in O C\left(X, \Im_{b}\right)$.

2. Let $x \in X$. Then, $b_{x} \in S P(X, A)$. As $\mathcal{M}$ is soft locally finite in $\left(X, \bigoplus_{a \in A} \Im_{a}, A\right)$, there is $F \in \bigoplus_{a \in A} \Im_{a}$ such that $b_{x}$ $\widetilde{\epsilon} F$, and $\left\{M \in \mathcal{M}: F \widetilde{\cap} M \neq 0_{A}\right\}$ is finite. Thus, $x \in F(b)$ $\in \Im_{b}$. If $M \in \mathcal{M}$ is such that $F(b) \cap M(b) \neq \emptyset$, then $F \widetilde{\cap}$ $M \neq 0_{A}$. It follows that $\mathcal{M}_{b}$ is locally finite in $\left(X, \Im_{b}\right)$.

3. Let $M(b) \in \mathcal{M}_{b}-\{\emptyset\}$, where $M \in \mathcal{M}$. As $\mathcal{M} \preceq \mathcal{H}$, there is $H \in \mathcal{H}$ such that $M \widetilde{\subseteq} H$, and thus $M(b) \subseteq H(b)$. As $M(b) \neq \emptyset$, we have $H(b) \neq \emptyset$, and thus there is $U \in \mathcal{U}$ such that $H=b_{U}$ and $H(b)=U$. It follows that $\mathcal{M}_{b} \preceq \mathcal{U}$.

Sufficiency. We assume that $\left(X, \Im_{a}\right)$ is paracompact for all $a \in A$. Let $\mathcal{B}=\left\{a_{Y}: a \in A\right.$ and $\left.Y \in \Im_{a}\right\}$. By Theorem 3.5 in [1], $\mathcal{B}$ is a soft base of $\bigoplus_{a \in A} \Im_{a}$. We apply Proposition 3.7. Let $\mathcal{H} \in S O C\left(X, \bigoplus_{a \in A} \Im_{a}, A\right)$ with $\mathcal{H} \subseteq \mathcal{B}$. For each $a \in A$, let $\mathcal{H}_{a}=\left\{Y \subseteq X: a_{Y} \in \mathcal{H}\right\}$. Then, for all $a \in A, \mathcal{H}_{a}$ $\in O C\left(X, \Im_{a}\right)$, where $\left(X, \Im_{a}\right)$ is paracompact, and thus there is $\mathcal{M}_{a} \in O C\left(X, \Im_{a}\right)$ such that $\mathcal{M}_{a}$ is locally finite in $\left(X, \Im_{a}\right)$ and $\mathcal{M}_{a} \preceq \mathcal{H}_{a}$. Let $\mathcal{G}=\left\{a_{Y}: a \in A\right.$ and $\left.Y \in \mathcal{M}_{a}\right\}$.

Claim. (1) $\mathcal{G} \in S O C\left(X, \bigoplus_{a \in A} \Im_{a}, A\right)$.

(2) $\mathcal{G}$ is soft locally finite in $\left(X, \bigoplus_{a \in A} \Im_{a}, A\right)$.

(3) $\mathcal{G} \preceq \mathcal{H}$.

Proof of Claim. 1. For all $a \in A$, we have $\mathcal{M}_{a} \subseteq \Im_{a}$, and therefore $\mathcal{G} \subseteq \bigoplus_{a \in A} \Im_{a}$. For all $a \in A$, we have $\mathcal{M}_{a} \in$ $O C\left(X, \Im_{a}\right)$, and thus $\left(\widetilde{\cup}\left\{a_{Y}: a \in A\right.\right.$ and $\left.\left.Y \in \mathcal{M}_{a}\right\}\right)(a)=$ $\cup\left\{Y: Y \in \mathcal{M}_{a}\right\}=X$. Therefore, $\widetilde{\cup}\left\{a_{Y}: a \in A\right.$ and $Y \in$ $\left.\mathcal{M}_{a}\right\}=1_{A}$. It follows that $\mathcal{G} \in S O C\left(X, \bigoplus_{a \in A} \Im_{a}, A\right)$.

2. Let $b_{x} \in S P(X, A)$. As $\mathcal{M}_{a}$ is locally finite in $\left(X, \Im_{b}\right)$, there is $O \in \Im_{b}$ such that $x \in O$, and $\{Y: O \cap Y \neq \emptyset\}$ is finite.

We have $b_{x} \in b_{O} \in \bigoplus_{a \in A} \Im_{a}$. If $b_{O} \widetilde{\cap} a_{Y} \neq 0_{A}$, then $a=b$ and $O \cap Y \neq \emptyset$. This shows that $\left\{a_{Y}: a \in A\right.$ and $Y \in \mathcal{M}_{a}$,

and $\left.b_{O} \widetilde{\cap} a_{Y} \neq 0_{A}\right\}$ is finite. It follows that $\mathcal{G}$ is soft locally finite in $\left(X, \bigoplus_{a \in A} \Im_{a}, A\right)$.

3. Let $a_{Y} \in \mathcal{G}$ with $a \in A$ and $Y \in \mathcal{M}_{a}$. As $\mathcal{M}_{a} \preceq \mathcal{H}_{a}$, there is $Z \in \mathcal{H}_{a}$, where $a_{Z} \in \mathcal{H}$, such that $Y \subseteq Z$. Therefore, $a_{Y} \widetilde{\subseteq} a_{Z}$. It follows that $\mathcal{G} \preceq \mathcal{H}$.

Proposition 3.9. Let $(X, \tau, A)$ be an STS, and let $\mathcal{B}$ be a soft base of $\tau_{\omega}$. Then, $(X, \tau, A)$ is soft $\omega^{*}$-paracompact if and only if for every $\mathcal{H} \in S O C\left(X, \tau_{\omega}, A\right)$ with $\mathcal{H} \subseteq \mathcal{B}$, there is $\mathcal{M} \in$ $\operatorname{SOC}\left(X, \tau_{\omega}, A\right)$ such that $\mathcal{M}$ is soft locally finite in $(X, \tau, A)$ and $\mathcal{M} \preceq \mathcal{H}$.

Proof. Straightforward.

Corollary 3.10. Let $(X, \tau, A)$ be an STS. Then, $(X, \tau, A)$ is soft $\omega^{*}$-paracompact if and only if for every $\mathcal{H} \in S O C\left(X, \tau_{\omega}, A\right)$ 
with $\mathcal{H} \subseteq\{F-H: F \in \tau$ and $H \in C S S(X, A)\}$, there is $\mathcal{M} \in S O C\left(X, \tau_{\omega}, A\right)$ such that $\mathcal{M}$ is soft locally finite in $(X, \tau, A)$ and $\mathcal{M} \preceq \mathcal{H}$.

Lemma 3.11. Let $(X, \tau, A)$ be an STS. If $\mathcal{B}$ is a soft base for $\tau$, then $\{B-H: B \in \mathcal{B}$ and $H \in C S S(X, A)\}$ is a soft base for $\tau_{\omega}$.

Proof. Straightforward.

Theorem 3.12. Let $(X, \tau, A)$ be an STS, where $A=\{a\}$. Then, $(X, \tau, A)$ is soft $\omega^{*}$-paracompact if and only if the TS $\left(X, \tau_{a}\right)$ is $\omega^{*}$-paracompact.

Proof. Necessity. We assume that $(X, \tau, A)$ is soft $\omega^{*}$-paracompact. Let $\mathcal{U} \in O C\left(X,\left(\tau_{a}\right)_{\omega}\right)$. By Proposition 2.9, we have $\left(\tau_{a}\right)_{\omega}=\left(\tau_{\omega}\right)_{a}$, and thus $\mathcal{U} \in O C\left(X,\left(\tau_{\omega}\right)_{a}\right)$.

Then, $\left\{a_{U}: U \in \mathcal{U}\right\} \in S O C\left(X, \tau_{\omega}, A\right)$. As $(X, \tau, A)$ is soft $\omega^{*}$-paracompact, there is $\mathcal{H} \in \operatorname{SOC}\left(X, \tau_{\omega}, A\right)$ such that $\mathcal{H}$ is soft locally finite in $(X, \tau, A)$ and $\mathcal{H} \preceq\left\{a_{U}: U \in \mathcal{U}\right\}$. Let $\mathcal{V}=\{H(a): H \in \mathcal{H}\}$.

Claim. (1) $\mathcal{V} \in O C\left(X,\left(\tau_{a}\right)_{\omega}\right)$.

(2) $\mathcal{V}$ is locally finite in $\left(X, \tau_{a}\right)$.

(3) $\mathcal{V} \preceq \mathcal{U}$.

Proof of Claim. 1. As $\mathcal{H} \subseteq \tau_{\omega}$, we have $\mathcal{V} \subseteq\left(\tau_{\omega}\right)_{a}$ and by Proposition 2.9, it follows that $\mathcal{V} \subseteq\left(\tau_{a}\right)_{\omega}$. In addition, as $\mathcal{H} \in S O C\left(X, \tau_{\omega}, A\right)$, we have $\widetilde{\cup}\{H: H \in \mathcal{H}\}=1_{A}$, and thus $(\widetilde{\cup}\{H: H \in \mathcal{H}\})(a)=\cup\{H(a): H \in \mathcal{H}\}=X$. It follows that $\mathcal{V} \in O C\left(X,\left(\tau_{a}\right)_{\omega}\right)$.

2. Let $x \in X$. Then, $a_{x} \in S P(X, A)$. As $\mathcal{H}$ is soft locally finite in $(X, \tau, A)$, there is $F \in \tau$ such that $a_{x} \widetilde{\in} F$, and $\left\{H \in \mathcal{H}: F \widetilde{\cap} H \neq 0_{A}\right\}$ is finite. Then, $x \in F(a) \in \tau_{a}$. Furthermore, if for $H \in \mathcal{H}$, we have $F(a) \cap H(a) \neq \emptyset$, then $F \widetilde{\cap} H \neq 0_{A}$. It follows that $\{H \in \mathcal{H}: F(a) \cap H(a) \neq \emptyset\}$ is finite. Therefore, $\mathcal{V}$ is locally finite in $\left(X, \tau_{a}\right)$.

3. Let $H(a) \in \mathcal{V}$, where $H \in \mathcal{H}$. As $\mathcal{H} \preceq\left\{a_{U}: U \in \mathcal{U}\right\}$, there is $U \in \mathcal{U}$ such that $H \subseteq a_{U}$; thus, $H(a) \subseteq U$. This shows that $\mathcal{V} \preceq \mathcal{U}$.

Sufficiency. We assume that $\left(X, \tau_{a}\right)$ is $\omega^{*}$-paracompact. Let $\mathcal{H} \in \operatorname{SOC}\left(X, \tau_{\omega}, A\right)$. Then, $\{H(a): H \in \mathcal{H}\} \in O C(X$, $\left.\left(\tau_{\omega}\right)_{a}\right)=O C\left(X,\left(\tau_{a}\right)_{\omega}\right)$. As $\left(X, \tau_{a}\right)$ is $\omega^{*}$-paracompact, there is $\mathcal{V} \in O C\left(X,\left(\tau_{a}\right)_{\omega}\right)=O C\left(X,\left(\tau_{\omega}\right)_{a}\right)$ such that $\mathcal{V}$ is locally finite in $\left(X, \tau_{a}\right)$ and $\mathcal{V} \preceq\{H(a): H \in \mathcal{H}\}$. Let $\mathcal{M}=$ $\left\{a_{V}: V \in \mathcal{V}\right\}$.

Claim. (1) $\mathcal{M} \in S O C\left(X, \tau_{\omega}, A\right)$.

(2) $\mathcal{M}$ is soft locally finite in $(X, \tau, A)$.

(3) $\mathcal{M} \preceq \mathcal{H}$.
Proof of Claim. 1. As $\mathcal{V} \subseteq\left(\tau_{\omega}\right)_{a}$, we have $\mathcal{M} \subseteq \tau_{\omega}$. In addition, as $\mathcal{V} \in O C\left(X,\left(\tau_{\omega}\right)_{a}\right)$, we have $\cup\{V: V \in \mathcal{V}\}=$ $X$, and thus $\widetilde{\cup}\left\{a_{V}: V \in \mathcal{V}\right\}=1_{A}$. It follows that $\mathcal{M} \in$ $\operatorname{SOC}\left(X, \tau_{\omega}, A\right)$.

2. Let $a_{x} \in S P(X, A)$. As $\mathcal{V}$ is locally finite in $\left(X, \tau_{a}\right)$, there is $O \in \tau_{a}$ such that $x \in O$ and $\{V \in \mathcal{V}: O \cap V \neq \emptyset\}$ is finite. Then, $a_{x} \tilde{\in} a_{O} \in \tau$. If $a_{V} \in \mathcal{M}$, where $V \in \mathcal{V}$ and $a_{O}$ $\widetilde{\cap} a_{V} \neq 0_{A}$, then $\left(a_{O} \widetilde{\cap} a_{V}\right)(a)=O \cap V \neq \emptyset$. It follows that $\mathcal{M}$ is soft locally finite in $\operatorname{SOC}(X, \tau, A)$.

3. Let $a_{V} \in \mathcal{M}$, where $V \in \mathcal{V}$. As $\mathcal{V} \preceq\{H(a): H \in \mathcal{H}\}$, there is $H \in \mathcal{H}$ such that $V \subseteq H(a)$, and thus $a_{V} \widetilde{\subseteq} H$. This shows that $\mathcal{M} \preceq \mathcal{H}$.

Theorem 3.13. Let $X$ be an initial universe, and let $A$ be a set of parameters. Moreover, let $\left\{\Im_{a}: a \in A\right\}$ be an indexed family of topologies on $X$. Then, $\left(X, \bigoplus_{a \in A} \Im_{a}, A\right)$ is soft $\omega^{*}$ paracompact if and only if $\left(X, \Im_{a}\right)$ is $\omega^{*}$-paracompact for all $a \in A$.

Proof. Necessity. We assume that $\left(X, \bigoplus_{a \in A} \Im_{a}, A\right)$ is soft $\omega^{*}$-paracompact. Let $b \in A$. To show that $\left(X, \Im_{b}\right)$ is $\omega^{*}$ paracompact, let $\mathcal{U} \subseteq O C\left(X,\left(\Im_{b}\right)_{\omega}\right)$. We set $\mathcal{H}=\left\{b_{U}: U \in\right.$ $\mathcal{U}\} \cup\left\{a_{X}: a \in A-\{b\}\right\}$. Then, $\mathcal{H} \in \operatorname{SOC}\left(X, \bigoplus_{a \in A}\left(\Im_{a}\right)_{\omega}, A\right)$. By Proposition 2.10, $\bigoplus_{a \in A}\left(\Im_{a}\right)_{\omega}=\left(\bigoplus_{a \in A} \Im_{a}\right)_{\omega}$, and thus $\mathcal{H} \in$ $\operatorname{SOC}\left(X,\left(\bigoplus_{a \in A} \Im_{a}\right)_{\omega}, A\right)$. As $\left(X, \bigoplus_{a \in A} \Im_{a}, A\right)$ is soft $\omega^{*}-$ paracompact, there is $\mathcal{M} \in \operatorname{SOC}\left(X,\left(\bigoplus_{a \in A} \Im_{a}\right)_{\omega}, A\right)$ such that $\mathcal{M}$ is soft locally finite in $\left(X, \bigoplus_{a \in A} \Im_{a}, A\right)$ and $\mathcal{M} \preceq \mathcal{H}$. By Proposition 2.10, $\mathcal{M} \in S O C\left(X, \bigoplus_{a \in A}\left(\Im_{a}\right)_{\omega}, A\right)$. Let $\mathcal{M}_{b}=\{M(b): M \in \mathcal{M}\}$.

Claim. (1) $\mathcal{M}_{b} \in O C\left(X,\left(\Im_{b}\right)_{\omega}\right)$.

(2) $\mathcal{M}_{b}$ is locally finite in $\left(X, \Im_{b}\right)$.

(3) $\mathcal{M}_{b} \preceq \mathcal{U}$.

Proof of Claim. 1. As $\mathcal{M} \subseteq \bigoplus_{a \in A}\left(\Im_{a}\right)_{\omega}$, by the definition of $\Im_{b}$, we have $\mathcal{M}_{b} \subseteq\left(\Im_{b}\right)_{\omega}$. As $\mathcal{M} \in \operatorname{SOC}\left(X, \bigoplus_{a \in A}\left(\Im_{a}\right)_{\omega}, A\right)$, we have $\widetilde{\cup}\{M: M \in \mathcal{M}\}=1_{A}$; hence, $(\widetilde{\cup}\{M: M \in \mathcal{M}\})(b)$ $=\cup\{M(b): M \in \mathcal{M}\}=X$. Therefore, $\mathcal{M}_{b} \in O C\left(X,\left(\Im_{b}\right)_{\omega}\right)$.

2. Let $x \in X$. Then, $b_{x} \in S P(X, A)$. As $\mathcal{M}$ is soft locally finite in $\left(X, \bigoplus_{a \in A} \Im_{a}, A\right)$, there is $F \in \bigoplus_{a \in A} \Im_{a}$ such that $b_{x}$ $\widetilde{\epsilon} F$, and $\left\{M \in \mathcal{M}: F \widetilde{\cap} M \neq 0_{A}\right\}$ is finite. Thus, $x \in F(b)$ 
$\in \Im_{b}$. If $M \in \mathcal{M}$ is such that $F(b) \cap M(b) \neq \emptyset$, then $F \widetilde{\cap}$ $M \neq 0_{A}$. It follows that $\mathcal{M}_{b}$ is locally finite in $\left(X, \Im_{b}\right)$.

3. Let $M(b) \in \mathcal{M}_{b}-\{\emptyset\}$, where $M \in \mathcal{M}$. As $\mathcal{M} \preceq \mathcal{H}$, there is $H \in \mathcal{H}$ such that $M \widetilde{\subseteq} H$, and thus $M(b) \subseteq H(b)$. As $M(b) \neq \emptyset$, we have $H(b) \neq \emptyset$, and thus there is $U \in \mathcal{U}$ such that $H=b_{U}$ and $H(b)=U$. It follows that $\mathcal{M}_{b} \preceq \mathcal{U}$.

Sufficiency. We assume that $\left(X, \Im_{a}\right)$ is $\omega^{*}$-paracompact for all $a \in A$. Let $\mathcal{B}=\left\{a_{Y}: a \in A\right.$ and $\left.Y \in\left(\Im_{a}\right)_{\omega}\right\}$. Then, $\mathcal{B}$ is a soft base of $\bigoplus_{a \in A}\left(\Im_{a}\right)_{\omega}=\left(\bigoplus_{a \in A} \Im_{a}\right)_{\omega}$. We apply Proposition 3.9. Let $\mathcal{H} \in S O C\left(X,\left(\bigoplus_{a \in A} \Im_{a}\right)_{\omega}, A\right)$ with $\mathcal{H} \subseteq \mathcal{B}$. For each $a \in A$, let $\mathcal{H}_{a}=\left\{Y \subseteq X: a_{Y} \in \mathcal{H}\right\}$. Then, for all $a \in A$, we have $\mathcal{H}_{a} \in O C\left(X,\left(\Im_{a}\right)_{\omega}\right)$, where $\left(X, \Im_{a}\right)$ is $\omega^{*}$-paracompact. Thus, there is $\mathcal{M}_{a} \in O C\left(X,\left(\Im_{a}\right)_{\omega}\right)$ such that $\mathcal{M}_{a}$ is locally finite in $\left(X, \Im_{a}\right)$ and $\mathcal{M}_{a} \preceq \mathcal{H}_{a}$. Let $\mathcal{G}=$ $\left\{a_{Y}: a \in A\right.$ and $\left.Y \in \mathcal{M}_{a}\right\}$.

Claim. (1) $\mathcal{G} \in \operatorname{SOC}\left(X,\left(\bigoplus_{a \in A} \Im_{a}\right)_{\omega}, A\right)$.

(2) $\mathcal{G}$ is soft locally finite in $\left(X, \bigoplus_{a \in A} \Im_{a}, A\right)$.

(3) $\mathcal{G} \preceq \mathcal{H}$.

Proof of Claim. 1. For all $a \in A$, we have $\mathcal{M}_{a} \subseteq\left(\Im_{a}\right)_{\omega}$, and therefore $\mathcal{G} \subseteq \bigoplus_{a \in A}\left(\Im_{a}\right)_{\omega}=\left(\bigoplus_{a \in A} \Im_{a}\right)_{\omega}$. For all $a \in$ $A$, we have $\mathcal{M}_{a} \in O C\left(X,\left(\Im_{a}\right)_{\omega}\right)$, and thus $\left(\widetilde{\cup}\left\{a_{Y}: a \in\right.\right.$ $A$ and $\left.\left.Y \in \mathcal{M}_{a}\right\}\right)(a)=\cup\left\{Y: Y \in \mathcal{M}_{a}\right\}=X$. Therefore, $\widetilde{\cup}\left\{a_{Y}: a \in A\right.$ and $\left.Y \in \mathcal{M}_{a}\right\}=1_{A}$. It follows that $\mathcal{G} \in$ $\operatorname{SOC}\left(X,\left(\bigoplus_{a \in A} \Im_{a}\right)_{\omega}, A\right)$.

2. Let $b_{x} \in S P(X, A)$. As $\mathcal{M}_{a}$ is locally finite in $\left(X, \Im_{b}\right)$, there is $O \in \Im_{b}$ such that $x \in O$, and $\{Y: O \cap Y \neq \emptyset\}$ is finite. We have $b_{x} \in b_{O} \in \bigoplus_{a \in A} \Im_{a}$. If $b_{O} \widetilde{\cap} a_{Y} \neq 0_{A}$, then $a=b$ and $O \cap Y \neq \emptyset$. This shows that $\left\{a_{Y}: a \in A\right.$ and $Y \in \mathcal{M}_{a}$, and $\left.b_{O} \widetilde{\cap} a_{Y} \neq 0_{A}\right\}$ is finite. It follows that $\mathcal{G}$ is soft locally finite in $\left(X, \bigoplus_{a \in A} \Im_{a}, A\right)$.

3. Let $a_{Y} \in \mathcal{G}$ with $a \in A$ and $Y \in \mathcal{M}_{a}$. As $\mathcal{M}_{a} \preceq \mathcal{H}_{a}$, there is $Z \in \mathcal{H}_{a}$, where $a_{Z} \in \mathcal{H}$, such that $Y \subseteq Z$. Therefore, $a_{Y} \widetilde{\subseteq} a_{Z}$. It follows that $\mathcal{G} \preceq \mathcal{H}$.

\section{Soft $\omega^{*}$-Paracompactness versus Soft Paracompactness}

Herein, we show by examples that soft $\omega^{*}$-paracompactness and soft paracompactness are independent of each other.
Theorem 4.1. Every soft regular soft $\omega^{*}$-paracompact STS is soft paracompact.

Proof. Let $(X, \tau, A)$ be soft regular and soft $\omega^{*}$-paracompact. Let $\mathcal{H} \in S O C(X, \tau, A) \subseteq S O C\left(X, \tau_{\omega}, A\right)$. Then, $\mathcal{H} \in$

$\operatorname{SOC}\left(X, \tau_{\omega}, A\right)$. As $(X, \tau, A)$ is soft $\omega^{*}$-paracompact, there is $\mathcal{M} \in \operatorname{SOC}\left(X, \tau_{\omega}, A\right)$ such that $\mathcal{M}$ is soft locally finite in $(X, \tau, A)$ and $\mathcal{M} \preceq \mathcal{H}$. It is clear that $\mathcal{M} \in S C(X, \tau, A)$. As $(X, \tau, A)$ is soft regular, by Theorem 5.14 in [37], it follows that $(X, \tau, A)$ is soft paracompact.

Theorem 4.2. If $(X, \tau, A)$ is a soft $\omega^{*}$-paracompact STS, then $\left(X, \tau_{\omega}, A\right)$ is soft paracompact.

Proof. We assume that $(X, \tau, A)$ is soft $\omega^{*}$-paracompact. Let $\mathcal{H} \in S O C\left(X, \tau_{\omega}, A\right)$. As $(X, \tau, A)$ is soft $\omega^{*}$-paracompact, there is $\mathcal{M} \in S O C\left(X, \tau_{\omega}, A\right)$ such that $\mathcal{M}$ is soft locally finite in $(X, \tau, A)$ and $\mathcal{M} \preceq \mathcal{H}$. As $\mathcal{M}$ is soft locally finite in $(X, \tau, A)$, we have that $\mathcal{M}$ is soft locally finite in $\left(X, \tau_{\omega}, A\right)$. It follows that $\left(X, \tau_{\omega}, A\right)$ is soft paracompact.

Definition 4.3. An STS $(X, \tau, A)$ is called strongly soft locally finite if for every $b_{x} \in S P(X, A)$, there exists $H \in \tau$ such that $\operatorname{Supp}(H)$ is finite, $b_{x} \widetilde{\in} H$, and $H(a)$ is finite for all $a \in A$.

Lemma 4.4. Let $(X, \tau, A)$ be a strongly soft locally finite STS, and let $\mathcal{H} \subseteq S P(X, A)$. Then, $\mathcal{H}$ is soft locally finite in $(X, \tau, A)$.

Proof. We assume that $(X, \tau, A)$ is a strongly soft locally finite, and let $\mathcal{H} \subseteq S P(X, A)$. Let $b_{x} \in S P(X, A)$. As $(X, \tau, A)$ is strongly soft locally finite, there exists $G \in \tau$ such that $\operatorname{Supp}(G)$ is finite, $b_{x} \widetilde{\in} G$, and $G(a)$ is finite for all $a \in A$. It is not difficult to verify that $\left\{a_{x}: a_{x} \widetilde{\in} G\right\}$ is finite. Thus, $\left\{H \in \mathcal{H}: G \widetilde{\cap} H \neq 0_{A}\right\}$ is finite. It follows that $\mathcal{H}$ is soft locally finite in $(X, \tau, A)$.

Theorem 4.5. Every strongly soft locally finite STS is soft $\omega^{*}$-paracompact.

Proof. We assume that $(X, \tau, A)$ is strongly soft locally finite. Let $\mathcal{H} \in S O C\left(X, \tau_{\omega}, A\right)$. Let $\mathcal{M}=S P(X, A)$. As $(X, \tau, A)$ is strongly soft locally finite, $(X, \tau, A)$ is soft locally countable, and by Corollary 14 in [2], we have that $\left(X, \tau_{\omega}, A\right)$ is a discrete STS. Thus, $\mathcal{M} \in \operatorname{SOC}\left(X, \tau_{\omega}, A\right)$. By Lemma $4.4, \mathcal{M}$ is soft locally finite in $(X, \tau, A)$. Moreover, it is clear that $\mathcal{M} \preceq \mathcal{H}$. It follows that $\left(X, \tau_{\omega}, A\right)$ is soft $\omega^{*}$-paracompact.

Theorem 4.6. Every strongly soft locally countable soft $\omega^{*}$-paracompact STS is strongly soft locally finite. 
Proof. Let $(X, \tau, A)$ be strongly soft locally countable and soft $\omega^{*}$-paracompact. Let $\mathcal{H}=S P(X, A)$. Then, by Corollary 14 in [2], $\left(X, \tau_{\omega}, A\right)$ is a discrete STS, and thus $\mathcal{H} \in$ $\operatorname{SOC}\left(X, \tau_{\omega}, A\right)$. As $(X, \tau, A)$ is soft $\omega^{*}$-paracompact, there is

$\mathcal{M} \in \operatorname{SOC}\left(X, \tau_{\omega}, A\right)$ such that $\mathcal{M}$ is soft locally finite in $(X, \tau, A), \mathcal{M} \preceq \mathcal{H}$, and for every $M \in \mathcal{M}$, we have $M \neq 0_{A}$. For every $M \in \mathcal{M}$, there is $a_{x_{M}}$ such that $M \widetilde{\subseteq} a_{x_{M}}$, and thus $M=a_{x_{M}}$. Therefore, $\mathcal{M} \subseteq S P(X, A)$. As $\mathcal{M} \in$ $S O C\left(X, \tau_{\omega}, A\right)$, we have $\mathcal{M}=S P(X, A)$. As $\mathcal{M}$ is soft locally finite in $(X, \tau, A)$, for every $a_{x} \in S P(X, A)$, there is $G \in \tau$ such that $a_{x} \widetilde{\in} G$, and $\left\{M \in \mathcal{M}: G \widetilde{\cap} M \neq 0_{A}\right\}=$ $\left\{b_{y} \in S P(X, A): G \widetilde{\cap} b_{y} \neq 0_{A}\right\}$ is finite; thus, $\operatorname{Supp}(G)$ is finite, and for every $b \in A, G(b)$ is finite. It follows that $(X, \tau, A)$ is strongly soft locally finite.

Theorem 4.7. If $(X, \tau, A)$ is soft separable and soft antilocally countable, then $\left(X, \tau_{\omega}, A\right)$ is not soft regular.

Proof. We assume toward a contradiction that $\left(X, \tau_{\omega}, A\right)$ is soft regular. As $(X, \tau, A)$ is soft separable, there is $G \in$ $\operatorname{SCSS}(X, A)$ such that $1_{A}=C l_{\tau}(G)$. As $(X, \tau, A)$ is strongly soft anti-locally countable, we have $1_{A} \notin S C S S(X, A)$; thus, $a_{x} \widetilde{\in} 1_{A}-G$. As $\left(X, \tau_{\omega}, A\right)$ is soft regular and $G$ is soft closed in $\left(X, \tau_{\omega}, A\right)$, there exist $U \in \tau_{\omega}, V \in \tau$ and $H \in C S S(X, A)$ such that $G \widetilde{\simeq} U, a_{x} \widetilde{\in} V-H$, and $U \widetilde{\cap}(V-H)=0_{A}$. As $1_{A}=C l_{\tau}(G)$ and $a_{x} \widetilde{\in} V \in \tau$, there is $b_{y} \widetilde{\in} G \widetilde{\cap} V$. As $b_{y}$ $\widetilde{\epsilon} G \widetilde{\subseteq} U$, there exist $W \in \tau$ and $M \in C S S(X, A)$ such that $b_{y} \widetilde{\in} W-M \widetilde{\subseteq} U$. As $b_{y} \widetilde{\in} U \widetilde{\cap} W \in \tau$, we have $U \widetilde{\cap}$ $W \in \tau-\left\{0_{A}\right\}$. As $(X, \tau, A)$ is soft anti-locally countable, we have $U \widetilde{\cap} W \notin C S S(X, A)$. As $U \widetilde{\cap} W-H \widetilde{\cap} G \widetilde{\subseteq} U$ $\widetilde{\cap}(V-H)=0_{A}$, we have $U \widetilde{\cap} W \widetilde{\subseteq} H \widetilde{\cap} G$, and thus $U \widetilde{\cap}$ $W \in C S S(X, A)$, which is a contradiction.

Theorem 4.8. Every soft anti-locally countable soft $T_{2}$ soft paracompact soft separable STS is not soft $\omega^{*}$-paracompact.

Proof. We assume that $(X, \tau, A)$ is soft anti-locally countable, soft $T_{2}$, soft $\omega^{*}$-paracompact, and soft separable. We assume toward a contradiction that $(X, \tau, A)$ is soft $\omega^{*}$-paracompact. As $(X, \tau, A)$ is soft $\omega^{*}$-paracompact, by Theorem $4.2\left(X, \tau_{\omega}, A\right)$ is soft paracompact. As $(X, \tau, A)$ is soft $T_{2}$, it is clear that $\left(X, \tau_{\omega}, A\right)$ is soft $T_{2}$. As $\left(X, \tau_{\omega}, A\right)$ is soft $T_{2}$ and soft paracompact, by Theorem 5.8 in [37], $\left(X, \tau_{\omega}, A\right)$ is soft regular. However, as $(X, \tau, A)$ is soft anti-locally countable and soft separable, by Theorem 4.1, $\left(X, \tau_{\omega}, A\right)$ is not soft regular, which is a contradiction.
Example 4.9. Let $A=\{a\}, X=\mathbb{R}$, and $\tau=\{F \in$ $S S(X, A): F(a)$ belongs to the usual topology on $\mathbb{R}\}$. Then, clearly, $(X, \tau, A)$ is soft anti-locally countable, soft $T_{2}$, soft paracompact, and soft separable. Thus, by Theorem $4.8,(X, \tau, A)$ is not soft $\omega^{*}$-paracompact.

Example 4.10. Let $(X, \Im)$ be the irrational slope topology (Example 75 in [38]). Let $A\{a\}$ and $\tau=\{F \in S S(X, A)$ : $F(a) \in \Im\}$. Clearly, $\left(X, \tau_{\omega}, A\right)$ is a discrete STS. However, by Theorem 4.6, $\left(X, \tau_{\omega}, A\right)$ is not soft $\omega^{*}$-paracompact.

\section{Conclusion}

We defined and studied a new concept STSs, soft $\omega^{*}$-para- compactness. Several characterizations and relationships regarding this new concept were proved. In the future, we intend to provide more characterizations and prove soft mapping theorems for soft $\omega^{*}$-paracompactness, as well as define new soft topological concepts using soft $\omega$-open sets.

\section{Conflict of Interest}

No potential conflict of interest relevant to this article was reported.

\section{References}

[1] S. Al Ghour and A. Bin-Saadon, "On some generated soft topological spaces and soft homogeneity," Heliyon, vol. 5, article no. e02061, 2019. https://doi.org/10.1016/j.heliyon. 2019.e02061

[2] S. Al Ghour and W. Hamed, "On two classes of soft sets in soft topological spaces," Symmetry, vol. 12, no. 2, article no. 265,2020 . https://doi.org/10.3390/sym12020265

[3] D. Molodtsov, "Soft set theory: first results," Computers and Mathematics with Applications, vol. 37, no. 4-5, pp. 19-31, 1999. https://doi.org/10.1016/S0898-1221(99) 00056-5

[4] M. Shabir and M. Naz, "On soft topological spaces," Computers and Mathematics with Applications, vol. 61, no. 7, pp. 1786-1799, 2011. https://doi.org/10.1016/j.camwa. 2011.02.006

[5] G. Oguz, "Soft topological transformation groups," Mathematics, vol. 8, no. 9, article no. 1545, 2020. https: //doi.org/10.3390/math8091545 
[6] V. Cetkin, E. Guner and H. Aygun, "On 2S-metric spaces," Soft Computing, vol. 24, pp. 12731-12742, 2020. https: //doi.org/10.1007/s00500-020-05134-w

[7] M. E. El-Shafei and T. M. Al-shami, "Applications of partial belong and total non-belong relations on soft separation axioms and decision-making problem," Computational and Applied Mathematics, vol. 39, article no. 138, 2020. https://doi.org/10.1007/s40314-020-01161-3

[8] J. C. R. Alcantud, "Soft open bases and a novel construction of soft topologies from bases for topologies," Mathematics, vol. 8, no. 5, article no. 672, 2020. https: //doi.org/10.3390/MATH8050672

[9] A. A. Bahredar and N. Kouhestani, "On $\varepsilon$-soft topological semigroups," Soft Computing, vol. 24, pp. 7035-7046, 2020. https://doi.org/10.1007/s00500-020-04826-7

[10] W. K. Min, "On soft $\omega$-structures defined by soft sets," International Journal of Fuzzy Logic and Intelligent Systems, vol. 20, no. 2, pp. 119-123, 2020. http://doi.org/10. 5391/IJFIS.2020.20.2.119

[11] T. M. Al-shami and M. E. El-Shafei, "Partial belong relation on soft separation axioms and decision-making problem, two birds with one stone," Soft Computing, vol. 24, no. 7, pp. 5377-5387, 2020. https://doi.org/10.1007/ s00500-019-04295-7

[12] T. M. Al-shami and M. E. El-Shafei, "T-soft equality relation," Turkish Journal of Mathematics, vol. 44, no. 4, pp. 1427-1441, 2020. https://doi.org/10.3906/mat-2005-117

[13] T. M. Al-shami and L. Kocinac, "Nearly soft Menger spaces," Journal of Mathematics, vol. 2020, article no. 3807418, 2020. https://doi.org/10.1155/2020/3807418

[14] T. M. Al-shami, L. Kocinac, and B. A. Asaad, "Sum of soft topological spaces," Mathematics, vol. 8, no. 6, article no. 990, 2020. https://doi.org/10.3390/math8060990

[15] T. M. Al-shami and M. E. El-Shafei, "Two new forms of ordered soft separation axioms," Demonstratio Mathematica, vol. 53, no. 1, pp. 8-26, 2020. https://doi.org/10.1515/ dema-2020-0002

[16] T. M. Al-shami and M. E. El-Shafei, "On supra soft topological ordered spaces," Arab Journal of Basic and Applied Sciences, vol. 26, no. 1, pp. 433-445, 2019. https://doi.org/10.1080/25765299.2019.1664101
[17] M. Kiruthika and P. Thangavelu, "A link between topology and soft topology," Hacettepe Journal of Mathematics and Statistics, vol. 48, no. 3, pp. 800-804, 2019. https: //doi.org/10.15672/HJMS.2018.551

[18] A. Al-Omari, "Soft topology in ideal topological spaces," Hacettepe Journal of Mathematics and Statistics, vol. 48, no. 5, pp. 1277-1285, 2019. https://doi.org/10.15672/ HJMS.2018.557

[19] T. M. Al-shami, M. E. El-Shafei, and M. Abo-Elhamayel, “On soft topological ordered spaces," Journal of King Saud University-Science, vol. 31, no. 4, pp. 556-566, 2019. https://doi.org/10.1016/j.jksus.2018.06.005

[20] N. C. Polat, G. Yaylali, and B. Tanay, "Some results on soft element and soft topological space," Mathematical Methods in the Applied Sciences, vol. 42, no. 16, pp. 56075614, 2019. https://doi.org/10.1002/mma.5778

[21] M. Abbas, G. Murtaza and S. Romaguera, "Remarks on fixed point theory in soft metric type spaces," Filomat, vol. 33, no. 17, pp. 5531-5541, 2019. https://doi.org/10.2298/ FIL1917531A

[22] T. M. Al-shami, M. E. El-Shafei, and M. Abo-Elhamayel, "Almost soft compact and approximately soft Lindelof spaces," Journal of Taibah University for Science, vol. 12, no. 5, pp. 620-630, 2018. https://doi.org/10.1080/ 16583655.2018 .1513701

[23] N. C. Polat, G. Yaylali, and B. Tanay, "A new approach for soft semi-topological groups based on soft element," Filomat, vol. 32, no. 16, pp. 5743-5751, 2018. https://doi. org/10.2298/FIL1816743P

[24] M. E. El-Shafei, M. Abo-Elhamayel, and T. M. Al-shami, "Partial soft separation axioms and soft compact spaces," Filomat, vol. 32, no. 13, pp. 4755-4771, 2018. https://doi. org/10.2298/FIL1813755E

[25] T. Y. Ozturk, C. G. Aras, and A. Yolcu, "Soft bigeneralized topological spaces," Filomat, vol. 32, no. 16, pp. 56795690, 2018. https://doi.org/10.2298/FIL18166790

[26] M. K. Tahat, F. Sidky, and M. Abo-Elhamayel, "Soft topological soft groups and soft rings," Soft Computing, vol. 22, no. 21, pp. 7143-7156, 2018. https://doi.org/10. 1007/s00500-018-3026-z 
[27] M. B. Kandemir, "The concept of $\sigma$-algebraic soft set," Soft Computing, vol. 22, no. 13, pp. 4353-4360, 2018. https://doi.org/10.1007/s00500-017-2901-3

[28] H. S. Al-Saadi and W. K. Min, "On soft generalized closed sets in a soft topological space with a soft weak structure," International Journal of Fuzzy Logic and Intelligent Systems, vol. 17, no. 4, pp. 323-328, 2017. https://doi.org/10.5391/IJFIS.2017.17.4.323

[29] H. Hdeib, “ $\omega$-closed mappings," Revista Colombiana de Matematicas, vol. 16, no. 1-2, pp. 65-78, 1982.

[30] S. Al Ghour and B. Irshedat, "On $\theta_{\omega}$ continuity," Heliyon, vol. 6, no. 2, article no. e03349, 2020. https://doi.org/10. 1016/j.heliyon.2020.e03349

[31] B. Roy, "On nearly Lindelof spaces via generalized topology," Proyecciones (Antofagasta), vol. 38, no. 1, pp. 49-57, 2019. https://doi.org/10.4067/S071609172019000100049

[32] P. Agarwal and C. K. Goel, "Delineation of $\Omega$ bitopological spaces," Proceedings of the Jangjeon Mathematical Society, vol. 22, no. 4, pp. 507-516, 2019.

[33] S. Al Ghour, "Theorems on strong paracompactness of product spaces," Mathematical Notes, vol. 103, pp. 54-58, 2018. https://doi.org/10.1134/S0001434618010066

[34] S. Al Ghour and K. Mansur, "Between open sets and semiopen sets," Universitas Scientiarum, vol. 23, no. 1, pp. 9-20, 2018. https://doi.org/10.11144/Javeriana.SC231.bosa
[35] S. Al Ghour and B. Irshedat, "The topology of $\theta_{\omega}$-open sets," Filomat, vol. 31, no. 16, pp. 5369-5377, 2017. https: //doi.org/10.2298/FIL1716369A

[36] S. Al Ghour, "On the product of meta Lindelof spaces," Far East Journal of Mathematical Sciences, vol. 4, no. 3, pp. 383-388, 2002.

[37] W. Rong and F. Lin, "Soft connected spaces and soft paracompact spaces," International Journal of Applied Mathematics and Statistics, vol. 51, no. 21, pp. 667-681, 2013.

[38] L. A. Steen and J. Seebach, Counterexamples in Topology, 1st ed. New York, NY: Holt, Rinehart and Winston, 1970.

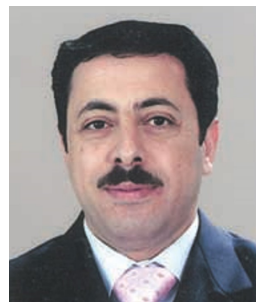

Samer Al Ghour received the Ph.D. in Mathematics from University of Jordan, Jordan in 1999. Currently, he is a professor at the Department of Mathematics and Statistics, Jordan University of Science and Technology, Jordan. His research interests include general topology, fuzzy topology, and soft set theory.

E-mail: algore@just.edu.jo 